\title{
DESERCION ESCOLAR Y CALIDAD DE LOS DOCENTES EN CHILE
}

\section{SCHOOL DROPOUT AND QUALITY OF TEACHERS IN CHILE}

\section{FELIPE SALCE DIAZ*}

\author{
Universidad de Atacama
}

\begin{abstract}
Resumen
Esta investigación analiza posibles causantes de la deserción escolar en alumnos de enseñanza secundaria en Chile entre 2004 y 2012. Se busca posibles causantes de la deserción escolar y se captura el efecto fijo de los docentes, para luego usarlo como índice de "valor agregado" y evaluar la movlidad de estos dentro del sistema educativo. Los primeros resultados muestran que los estudiantes de establecimientos municipales o que hayan reprobado anteriormente tienen mayores probabilidades de deserción. Por otro lado, se observa que los profesores con menor valor agregado tienen mayores probabilidades de trabajar en establecimientos municipales y de abandonar establecimientos particulares.
\end{abstract}

Palabras clave: Educación, valor agregado, valor añadido, abandono escolar.

Clasificación JEL: I21, I24, I29.

* Académico del Departamento de Ingeniería Comercial de la Universidad de Atacama, Chile.

Master of Arts in Economics de Georgetown University y Magister en Economia de la Universidad Alberto Hurtado. E-mail: felipe.salce@uda.cl

Las opiniones expresadas en este documento y los errores subsistentes son de exclusiva responsabilidad del autor. El autor agradece los valiosos comentarios de Eugenio Giolito y los revisores anónimos de Revista de Análisis Económico. 


\begin{abstract}
This research analyzes possible causes of school desertion in high school students in Chile between 2004 and 2012. It looks for possible causes of school dropout and captures the fixed effect of teachers, to then use it as an index of "added value" and evaluate the movement of teachers within the education system. The first results show that students from municipal schools or those who have previously failed are more likely to drop out. On the other hand, it is observed that teachers with lower added value are more likely to work in municipal establishments and to leave private establishments.
\end{abstract}

Keywords: Education, value-added, school dropout.

JEL Classification: I21, I24, I29.

\title{
1. INTRODUCCION
}

Mucho se ha discutido en Chile en los últimos años sobre la calidad de la educación y de los factores que pueden afectar en el rendimiento académico de los alumnos. Factores relacionados con la calidad de los docentes, factores socioeconómicos y/o factores de desempeño académico previo del estudiante en sí mismo. Sin embargo, un tema que ha pasado desapercibido en Chile es la deserción escolar, invisibilizando y minimizando el problema.

Pese a que el derecho a la educación está constitucionalmente resguardado, en Chile actualmente existe una serie de problemas relacionados con la calidad y el acceso a la educación, dejando fuera del sistema educativo a miles de niños y jóvenes cada año.

La educación en Chile se divide en cuatro etapas: (i) educación parvularia, (ii) educación básica o primaria, (iii) educación media o secundaria, y (iv) educación superior. De estas cuatro etapas las tres primeras son obligatorias ${ }^{123}$. Este trabajo se centra en la educación primaria y en la educación secundaria.

El nivel básico o primario tiene una duración de ocho años; de $1^{\circ}$ a $8^{\circ}$ básico, y el nivel medio o secundario tiene una duración de cuatro años; de $1^{\circ}$ a $4^{\circ}$ medio. Dentro de estos niveles es donde ocurre la deserción escolar, el cual es el fenómeno dónde el estudiante decide abandonar el sistema educativo antes de la edad mínima

\footnotetext{
Ley 19.876 del año 2003.

Para la educación parvularia, solo el último año (kinder) es obligatorio.

La educación chilena está regida por la Ley General de Educación del año 2009, donde los tres primeros niveles están supervisados por el Ministerio de Educación.
} 
establecida por la ley, y por ende no completa su educación obligatoria. Este hecho en particular produce tanto costos a nivel social como a nivel privado.

Dentro de los costos sociales están incluidos los mayores gastos que deben hacer los gobiernos en programas sociales que apuntan a las personas que no tienen la capacidad de generar ingresos laborales por sí mismos, donde están las personas con un bajo nivel educativo. Por otro lado la deserción escolar tiene un impacto negativo sobre el nivel de capital humano, lo cual reduce la tasa de crecimiento de la economía (Barro, 1991; Mankiw, Romer y Weil, 1992). También es importante considerar el aumento en el desempleo juvenil, y posterior desempleo adulto, al tener personas con pocos años de educación.

Por otro lado, los costos privados están vinculados principalmente a la disminución en los ingresos laborales las personas que abandonan el sistema educativo sin finalizarlo y reducción en la probabilidad de encontrar empleo. Según estimaciones realizadas en el estudio de CEPAL (2002), para el caso de Chile, un aumento de dos años en la escolaridad, aumentaría el ingreso laboral en un $23 \%$ para hombres y $25 \%$ para mujeres en zonas urbanas.

Al estimar la probabilidad de que los alumnos deserten del sistema educativo, y usando los controles adecuados, podemos capturar el efecto fijo de los profesores en la decisión de deserción de los alumnos, al que llamaremos el valor agregado del docente o profesor. Finalmente usamos este valor agregado para explicar en qué tipo de establecimientos educacionales trabajan y la movilidad que tienen estos, es decir, si tienden a quedarse en algún tipo de establecimiento o moverse entre las diferentes categorías de establecimientos educacionales. Si bien la metodología no es del todo novedosa, ésta no ha sido ocupada la decisión de deserción de los estudiantes, ni tampoco utilizada en otros aspectos para el caso de Chile como en países más desarrollados.

Este trabajo contribuye a la literatura al analizar el impacto de los posibles causantes de la deserción escolar, no solo del ámbito socioeconómico como es lo usual, sino también causantes dentro del mismo ámbito académico usando fuentes oficiales del Estado. Además se hace un aporte a la reducida literatura sobre el valor agregado de los profesores en Chile, capturando su relación con la decisión de deserción escolar de los alumnos, y luego contrastando este índice de valor agregado con la movilidad que tienen los docentes dentro del sistema educativo en el país.

Siguiendo la idea de Chetty, Friedman y Rockoff (2014a), desarrollamos una metodología para estimar el valor agregado de los docentes o profesores. En dicho trabajo, los autores estiman el valor agregado de los profesores a través en la diferencia en los exámenes estandarizados de los alumnos en años consecutivos, asumiendo que el cambio en el rendimiento del alumno es atribuible al profesor responsable. Por otro lado, en este trabajo dicho valor agregado está basado en el efecto fijo de los mismos profesores cuando analizamos la deserción escolar de los alumnos. Es importante mencionar que para realizar este procedimiento debemos asumir, al igual que Chetty, Friedman y Rockoff (2014a) y cualquier metodología de valor agregado, que los 
profesores se asignan de manera aleatoria entre los distintos grupos de alumnos, lo cual puede ser un supuesto discutible.

Los resultados muestran que los profesores con menor valor agregado tienen mayores probabilidades de trabajar en establecimientos municipales pero menos probabilidades de trabajar en establecimientos particulares. De la misma forma, se observa que los docentes de mayor valor agregado tienen mayores probabilidades de trabajar en establecimientos privados y menos probabilidades de trabajar en establecimientos municipales. Además se observa que los profesores de menor valor agregado tienden a abandonar más fácilmente establecimientos particulares, pero tienden a quedarse en establecimientos municipales, y por el contrario, los docentes de mayor valor agregado tienden a quedarse en establecimientos privados, pero salen de establecimientos municipales.

El documento está estructurado de la siguiente manera. En la sección 2 se hace una breve revisión de la literatura asociada a la deserción escolar y a medición de calidad docente. En la sección 3 se presenta la estrategia empírica utilizada. En la sección 4 se explican los datos que se usan en el trabajo y se presentan algunas estadísticas descriptivas. En la sección 5 se presentan los principales resultados de la investigación. Por último, en la sección 6 se muestran las conclusiones del trabajo.

\section{REVISION DE LA LITERATURA}

Si bien no existe literatura que estudie el problema de la deserción escolar con algún índice de calidad docente o su valor agregado, si hay bastantes investigaciones en ambos temas por separado. Sobre el abandono o deserción escolar hay investigaciones tanto para países desarrollados como Estados Unidos y países de Europa, como para países menos desarrollados como el caso de Chile y otros países latinoamericanos. Por otro lado, en temas de calidad docente la literatura es más reducida y generalmente proveniente de Estados Unidos. Estos trabajos aplican metodologías difíciles de replicar en Chile debido a las diferencias de los sistemas educativos y de la periodicidad de sus evaluaciones estandarizadas, lo cual limita los trabajos posibles en el país con estás metodologías.

Los principales aportes sobre deserción escolar para el caso chileno son de Filgueira, Fuentes y Filgueira. (2001), Melis, Díaz y Palma (2005) y Santos (2009). Estos trabajos estiman la probabilidad de deserción escolar usando modelos de variable binaria buscando sus posibles causas socioeconómicas. Por otro lado, los trabajos de Beyer (1998) y Sapelli y Torche (2004) analizan la deserción escolar en paralelo con el desempleo juvenil. Finalmente, Montero (2007) usa una metodología similar a Sapelli y Torche (2004) para analizar la deserción escolar obteniendo resultados similares en cuanto a la deserción escolar.

En general, estos autores llegan a conclusiones similares. Ellos muestran que existe una relación directa entre la probabilidad de deserción escolar y ciertas características 
socioeconómicas del alumno. Demuestran que cuando los alumnos tienen padres de menores estudios, o bajos niveles de ingresos per cápita en el hogar, hay una mayor probabilidad de deserción escolar. También encuentran una relación positiva entre la probabilidad de abandono escolar con características tales como que el alumno sea hombre, tenga un hijo, o que el alumno no viva con sus padres.

El problema de los estudios anteriores es que ocupan datos de encuestas de hogares ${ }^{4}$, las cuales obtienen resultados auto reportados que no otorgan una total confianza, ya que siempre hay un sesgo al reportar ingresos y nivel educacional, y otras situaciones de mayor complejidad que no quedan reflejadas (situaciones de abuso u otras prácticas ilegales las cuales los entrevistados tienden a omitir). Por otro lado, se entrevista a las personas en edades muy superiores al momento de la deserción.

Rodríguez et al. (2016) en lugar de centrarse únicamente en buscar los determinantes de la deserción escolar, se centran también en la predicción de la deserción escolar. Para ello ocupan un algoritmo de clasificación, en la línea de aprendizaje automático (comúnmente llamado Machine Learning en la literatura) y concluyen que existe un espacio para continuar con esta línea de trabajo, aunque para ello se requiere una mayor cantidad de datos relevantes, tales como sus calificaciones y asistencia, además de mayor coordinación y dialogo entre las agencias en el diseño de políticas públicas con respecto a este tema. Al igual que los autores anteriores, Rodríguez et al. (2016) utiliza encuestas de hogares que tienen las falencias ya mencionadas, además de no tener acceso a variables como las calificaciones y asistencia, variables que si se tienen en bases de datos oficiales del Ministerio de Educación.

Las investigaciones recientemente desarrolladas sobre el nivel de productividad de los docentes se basan en modelos de "valor agregado" para estimar el impacto de los profesores en el rendimiento de los estudiantes. Los modelos que usan el valor agregado de los profesores en sus estimaciones se han vuelto populares en los planes de investigación en el último tiempo.

Siguiendo esta línea, el trabajo Hill, Kapitula y Umland (2011) encuentra que el valor agregado de los docentes de matemática está directamente relacionado con su conocimiento propio del área y con el número de alumnos a los que enseñan. Por otro lado, en la investigación de Sass, Semykina y Harris (2014) desarrollaron seis modelos distintos de valor agregado a partir de un modelo estructural de rendimiento estudiantil usando datos del estado de Florida para comparar la sensibilidad de sus estimaciones. El trabajo de Cavicchiolo, Alivernini y Manganelli (2015) realiza algo parecido pero para estimar el valor agregado de los métodos de enseñanza. Los autores en su trabajo exploran las relaciones entre las mejoras en las calificaciones en matemática de los alumnos y los métodos de enseñanza utilizados por sus profesores que participaron de un programa especial para mejorar la enseñanza y el aprendizaje

4 En Chile la encuesta de hogares más común es la Encuesta de Caracterización Socioeconómica Nacional (CASEN) 
en Italia. En la investigación de Koedel, Mihaly y Rockoff (2015) se revisa la literatura existente sobre el valor agregado de los profesores cubriendo temas que van desde los aspectos técnicos del diseño del modelo hasta el uso del valor agregado en las políticas públicas. Ellos encuentran una gran consistencia en los resultados y conclusiones de los distintos trabajos revisados, concluyendo que la literatura en esta área está convergiendo a resultados similares.

En esta misma línea, el principal aporte seguido en esta investigación es el trabajo de Chetty, Firedman y Rockoff (2014a). En dicho trabajo, los autores se basan en atribuir al docente las diferencias en los resultados de exámenes de dos años consecutivos. Ellos encuentran que los alumnos que están con profesores de mayor valor agregado tienen mejores resultados en estos exámenes. Además en Chetty, Firedman y Rockoff (2014b) encuentran efectos de largo plazo en una mejor asistencia a la universidad y en mayores ingresos al haber estado con docentes de mayor valor agregado. En el trabajo de Canales y Maldonado (2018) se realiza un procedimiento similar para el caso de Chile, con alumnos que rinden la prueba Simce en su último año de primaria para los años 2007 y 2011.

\section{METODOLOGIA}

En este estudio, se ha buscado obtener, a través de un modelo simple y con la información disponible: (i) la importancia y las magnitudes de las posibles causas de la deserción escolar en $1^{\circ}$ medio, y (ii) evaluar a los profesores según su participación en el abandono escolar de sus estudiantes. Para este estudio se ha observado a todos los alumnos entre los cohortes 2004 y 2012, y se ha evaluado si han desertado o no del sistema educativo estando en $1^{\circ}$ medio, observándolos incluso hasta el año 2016.

Siguiendo la línea de estimar el valor agregado de los docentes, primero se estima la probabilidad de deserción escolar de los alumnos, controlando, entre otras variables, por los profesores que tuvieron. En esta estimación capturamos el efecto fijo de los docentes y lo llamamos nuestro "valor agregado", para posteriormente usarlo como variable explicativa sobre posibles los movimientos de los profesores entre distintos tipos de establecimientos educativos. Se busca explicar en qué tipo de establecimiento educativo hacen o dejan de realizar clases los profesores según su nivel de valor agregado en la deserción escolar de sus estudiantes.

Para temas de educación en Chile, la información disponible es bastante amplia, tanto en diferentes tipos de datos como en la cantidad de años registrados, comenzando a estar disponible desde la década de 1990 en muchos casos. Sin embargo, en Chile no existe una prueba estandarizada que rindan todos los alumnos todos los años como en países más desarrollados. Lo más parecido en Chile es la prueba Simce que es todos 
los años, pero únicamente para ciertos grados académicos. Esta prueba proporciona una idea del alumno y, en algunos alumnos, sus características socioeconómicas proveniente de un cuestionario a los padres o tutores. Dado que la prueba Simce los alumnos no la dan todos los años, y en algunos casos una sola vez en su vida, se debe usar algún instrumento para reemplazar a la prueba Simce, y así no perder observaciones. Como instrumento de rendimiento académico previo de los alumnos, en este trabajo se utilizan efectos fijos por el establecimiento educativo al que alumno asistió en $8^{\circ}$ básico, y el decil de notas en el que se encuentra el alumno dentro de su establecimiento en el mismo grado. Usando estas variables en conjunto nos proporciona una información bastante certera sobre la situación socioeconómica de alumno y su rendimiento académico.

\section{a) Modelo Econométrico}

Para este análisis, primero se estima un modelo utilizando la información disponible del alumno $i$ y su establecimiento $s$ tanto en $1^{\circ}$ medio como en $8^{\circ}$ básico, además de los profesores $j$ que tuvo en $1^{\circ}$ medio. En este primer modelo, se han utilizado los efectos fijos por establecimiento de $8^{\circ}$ básico y el decil del alumno dentro de su propio establecimiento en ese grado. Además se ha utilizado como controles para el establecimiento de $1^{\circ}$ medio si es rural o no. También se han utilizado los siguientes controles para el alumno: (i) género, (ii) si repitió en la enseñanza básica, (iii) si se cambió de establecimiento entre $8^{\circ}$ básico y $1^{\circ}$ medio, y (iv) el número de veces que ha cursado $1^{\circ}$ medio.

El modelo econométrico utilizado para estimar la deserción escolar en $1^{\circ}$ medio puede representarse a través de la Ecuación 1.

$$
d_{i j s t}=\alpha_{1}+\beta_{1}^{\prime} \boldsymbol{X}_{1 i}+\beta_{2}^{\prime} \boldsymbol{X}_{2 s}+\delta_{j}+\gamma_{s} \cdot \lambda_{i s}+\theta_{t}+\varepsilon_{i j s t}
$$

donde $d_{i j s t}$ es una variable binaria igual a uno si el alumno $i$ desertó del establecimiento educativo $s$, con el profesor $j$ en $1^{\circ}$ medio en el año $t$. $\boldsymbol{X}_{1 i}$ es el vector de controles del alumno $i . X_{2 s}$ es el vector de controles del establecimiento $s$ donde el alumno cursó $1^{\circ}$ medio. $\delta_{j}$ son los efectos fijos por cada profesor $j$ que tuvo el alumno $i$ en $1^{\circ}$ medio $^{5} . \lambda_{s}$ son variables binarias por cada establecimiento $s$ de $8^{\circ}$ básico y $\lambda_{i s}$ son variables binarias según el ranking del alumno $i$ dentro del establecimiento $s$ cuando cursó $8^{\circ}$ básico. Finalmente $\theta_{t}$ son variables binarias por año.

En esta primera estimación se captura el efecto fijo de los profesores y posteriormente se ordena en cuartiles según la magnitud de su efecto fijo o valor agregado estimado.

5 En nuestro modelo solo consideramos los profesores de matemática y lenguaje, por ende cada alumno tiene únicamente dos profesores como máximo en cada año. 
Esto es usado en una segunda estimación como variable explicativa para estimar distintas variables de movilidad de los profesores. Para esta segunda estimación se crea un panel de datos de profesores por año desde el 2004 hasta el 2015. Además de considerar el efecto fijo de los docentes ya mencionado, se han utilizado distintos controles: (i) edad, (ii) años de experiencia, (iii) sexo, (iv) si estudió en una universidad, o por el contrario, en otro tipo de institución, y (v) si sus estudios fueron en modalidad presencial o no. Usando estos controles se busca evidencia empírica que demuestre que el efecto fijo de los profesores, o su valor agregado, tiene alguna relación con el tipo de establecimiento en el que están los profesores, o si tienden a moverse entre distintos tipos de establecimientos educacionales. Finalmente el modelo a estimar se puede representar en la Ecuación 2:

$$
y_{j t}=\alpha_{2}+\pi^{\prime} \boldsymbol{X}_{j}+\theta_{t}+\varepsilon_{j t}
$$

donde $y_{j t}$ es la variable dependiente de cada modelo para el profesor $j$ en el año $t . \boldsymbol{X}_{\boldsymbol{j}}$ es el vector de controles ya mencionados del profesor $j$, donde está incluido el cuartil al que pertenece $\delta_{j}$ de la ecuación 1 y $\theta_{t}$ son variables binarias por año.

Finalmente, las variables dependientes a estimar son las siguientes: (i) probabilidad que el profesor $j$ haga clases en el tipo de establecimiento $k$, (ii) probabilidad que el profesor $j$ haga clases en el tipo de establecimiento $k$, y que únicamente haga clases en ese tipo de establecimiento, (iii) probabilidad que el profesor $j$ haga clases en el tipo de establecimiento $k$ en el año $t$, dado que realizaba clases en el mismo tipo de establecimiento $k$ en el año $t-1$, (iv) probabilidad que el profesor $j$ abandone el establecimiento tipo $k$. Donde el tipo del establecimiento $k$, puede ser un establecimiento municipal, particular subvencionado o particular no subvencionado.

\section{DATOS}

Los datos que usamos en esta investigación son datos administrativos oficiales del Ministerio de Educación de Chile. Se recopilan datos de estudiantes, establecimientos educativos y docentes. Usando estos datos, podemos identificar el establecimiento educativo $s$ al que asistía el estudiante $i$ en cada año $t$. También podemos identificar a los docentes $j$ que le hacían clases al estudiante $i$ en cada año. En todos los casos los datos son obtenidos del Centro de Estudios del Ministerio de Educación entre los años 2004 y 2016.

\section{a) Establecimientos Educacionales}

En el caso de los Establecimientos Educacionales, los datos nos permiten diferenciar a cada establecimiento en cada año. Las características más relevantes de 
los establecimientos es su dependencia administrativa, es decir, si es un establecimiento municipal, particular subvencionado, o particular no subvencionado, y si es un establecimiento rural o urbano ${ }^{6}$.

En Chile hay cuatro tipos de establecimientos educacionales: (i) establecimientos municipales, (ii) establecimientos particulares subvencionados, (iii) establecimientos particulares no subvencionados, y (iv) establecimientos llamados corporación de administración delegada.

Los establecimientos municipales pertenecen al Estado, el cual delega su administración en las municipalidades y les entrega financiamiento para que se hagan responsables de estos establecimientos. Este tipo de financiamiento se denomina subvención escolar. Los establecimientos particulares subvencionados son de propiedad privada pero también reciben financiamiento del Estado a través de subvenciones escolares, además de tener la posibilidad de cobrar una matrícula y/o mensualidad a sus alumnos (financiamiento compartido). Los establecimientos particulares no subvencionados son de propiedad privada y no reciben ningún tipo de subvención por parte del Estado. Los establecimientos denominados corporación de administración delegada pertenecen al Estado pero la administración fue entregada a distintas corporaciones y fundaciones sin fines de lucro en 1980.

En el año 2016 había 6.805 establecimientos municipales representando al 42,6\% del total de establecimientos, 7.604 establecimientos particulares subvencionados representando al 47,6\%, 1.511 establecimientos particulares no subvencionados representando al 9,4\% y 70 establecimientos de administración delegada representando menos del 0,3\% del total de establecimientos en el país. En el mismo año, el 35,9\% de los alumnos estudiaba en un establecimiento municipal, el 54,7\% en un establecimiento particular subvencionado, un $8,1 \%$ en un establecimiento particular no subvencionado, y el 1,3\% en un establecimiento de Administración Delegada ${ }^{7}$. La evolución de estos porcentajes en los últimos años puede observarse en la Figura 1.

6 Unicamente se han usado establecimientos de educación convencional, es decir, se han excluido los establecimientos educación especial, educación para adultos y ramas militares.

7 Debido a la poca partición de los establecimientos educacionales de administración delegada, se han excluido de esta investigación. Si bien no se han eliminado para los cálculos y análisis, los resultados en cuanto a éste tipo de establecimientos no son mostrados. 


\section{FIGURA 1}

\section{EVOLUCION DE LA COMPOSICION DE LA MATRICULA SEGUN DEPENDENCIA ADMINISTRATIVA}

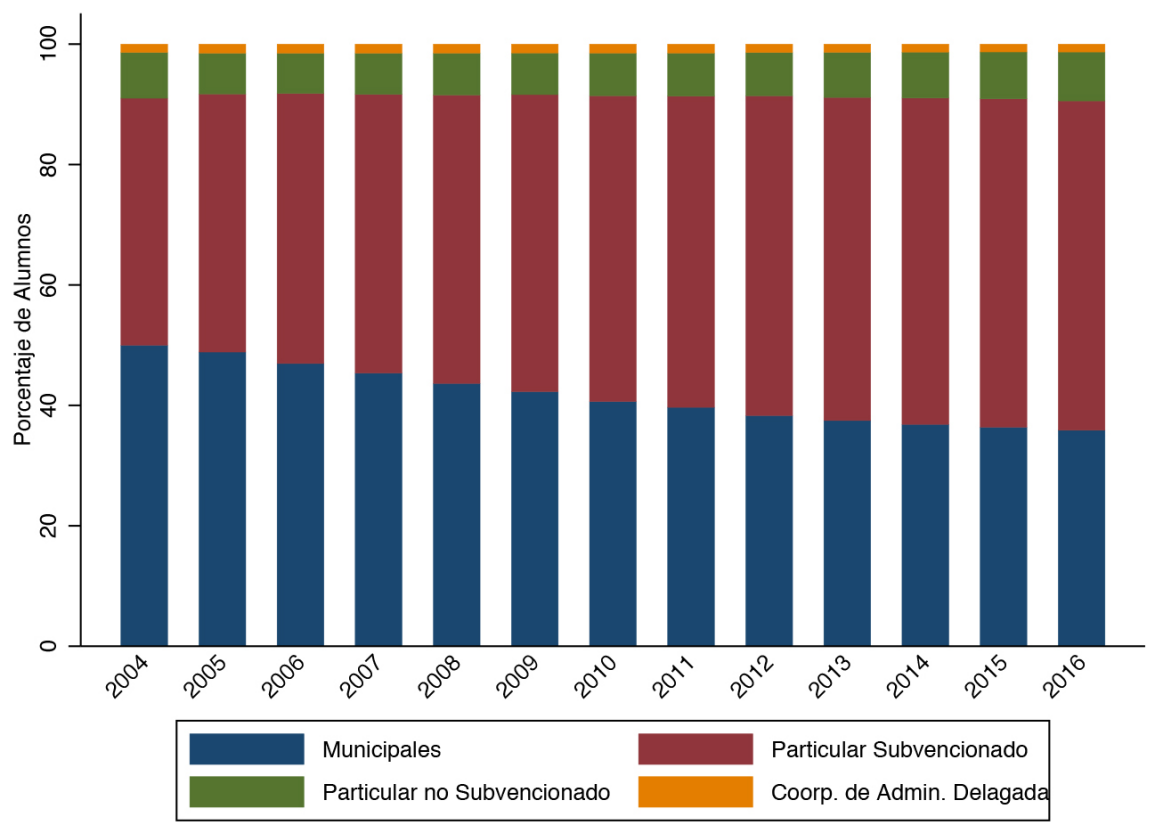

Fuente: Elaboración propia en base a datos de Centro de Estudios, Ministerio de Educación. Chile.

Además de la separación por su dependencia administrativa, los establecimientos pueden ser rurales o urbanos. Para el año 2016 el 92,4\% de los establecimientos era urbano, mientras que el 7,6\% restante era rural.

\section{b) Alumnos}

En el caso de los alumnos, los datos entregan las características académicas de los alumnos, como su porcentaje de asistencia cada año, su calificación final de cada año y si aprobó o no el año académico.

Al tener datos de panel para los alumnos los observamos durante varios años consecutivos, y en algunos casos en particular, durante su vida escolar completa. Uniendo las base de datos de cada año podemos observar si el alumno se ha cambiado o no de establecimiento o, por ejemplo, si ha repetido algún año escolar o a abandonado 
el sistema educativo. En la Tabla 1 se muestran algunas estadísticas descriptivas de los alumnos de la muestra completa.

\section{TABLA 1}

ESTADISTICAS DESCRIPTIVAS DE LOS ALUMNOS

PARA LA MUESTRA USADAS

\begin{tabular}{|c|c|c|}
\hline Variable & $\begin{array}{c}\text { Media } \\
\text { (1) }\end{array}$ & $\begin{array}{c}\text { Observaciones } \\
\text { (2) }\end{array}$ \\
\hline Hombres & $50,04 \%$ & 2.750 .220 \\
\hline Se cambiaron de establecimiento & $67,77 \%$ & 2.750 .220 \\
\hline Reprobaron algún año en primaria & $12,50 \%$ & 2.750 .220 \\
\hline $\mathrm{N}^{\circ}$ de veces que cursaron $1^{\circ}$ medio & 1,14 & 2.750 .220 \\
\hline Promedio General $1^{\circ}$ medio & 5,31 & 2.673 .522 \\
\hline Asistencia en $1^{\circ}$ medio & $91,88 \%$ & 2.673 .522 \\
\hline Promedio General $8^{\circ}$ básico & 56,05 & 2.750 .220 \\
\hline Asistencia en $8^{\circ}$ básico & $93,66 \%$ & 2.750 .197 \\
\hline Deserción & $7,52 \%$ & 2.750 .220 \\
\hline
\end{tabular}

Fuente: Elaboración propia en base a datos de Centro de Estudios, Ministerio de Educación. Chile.

\section{c) Profesores o Docentes}

En el caso de los docentes, la base de datos entrega información personal como su género, edad, y años de experiencia laboral. Por otro lado, los datos también entregan su información profesional como el tipo de institución donde estudió (Universidad, Centro de Formación Técnica, Instituto Profesional, Escuela Normal u otro tipo de institución), años de duración de la carrera y si fueron estudios presenciales o no. En este trabajo solamente se ocupan los profesores de Matemática y Lenguaje.

Al igual que en el caso de los estudiantes, al observar a los profesores durante un largo período de tiempo nos permite saber si han sido contratados en nuevos establecimientos educacionales, o si han salido de ellos. También es importante para nuestro análisis saber en cuantos establecimientos educacionales por año estuvieron los profesores.

Finalmente, en la Tabla 2 se muestran algunas estadísticas descriptivas de los docentes de la muestra completa, en la Tabla 3 se muestran estadísticas descriptivas del efecto fijo de los profesores y en la Figura 2 se pueden observar dos histogramas de este efecto fijo. 
TABLA 2

ESTADISTICAS DESCRIPTIVAS DE LOS PROFESORES PARA LA MUESTRA USADA

\begin{tabular}{|l|c|c|}
\hline \multicolumn{1}{|c|}{ Variable } & $\begin{array}{c}\text { Media } \\
(1)\end{array}$ & $\begin{array}{c}\text { Observaciones } \\
(2)\end{array}$ \\
\hline Hombres & $38,59 \%$ & 23.966 \\
Experiencia & 12,06 & 21.573 \\
Edad & 36,12 & 23.961 \\
Número de establecimientos por año & 1,36 & 23.966 \\
Estudiaron en Universidad & $97,28 \%$ & 23.966 \\
Estudios Presenciales & $97,75 \%$ & 21.548 \\
\hline
\end{tabular}

Fuente: Elaboración propia en base a datos de Centro de Estudios, Ministerio de Educación. Chile.

TABLA 3

ESTADISTICAS DESCRIPTIVAS DEL EFECTO FIJO DE LOS PROFESORES

\begin{tabular}{|l|c|}
\hline \multicolumn{1}{|c|}{ Estadístico } & $(1)$ \\
\hline Promedio & 0,10165 \\
Desviación Estándar & 0,04351 \\
Mínimo & $-0,55127$ \\
Máximo & 1,04216 \\
Observaciones & 24.568 \\
& \\
Percentil 1\% & 0,0083 \\
Percentil 5\% & 0,0601 \\
Percentil 10\% & 0,0772 \\
Percentil 25\% & 0,0917 \\
Percentil 50\% & 0,1007 \\
Percentil 75\% & 0,1098 \\
Percentil 90\% & 0,1230 \\
Percentil 95\% & 0,1361 \\
Percentil 99\% & 0,2083 \\
\hline
\end{tabular}

Fuente: Elaboración propia en base a datos de Centro de Estudios, Ministerio de Educación. Chile. 


\section{FIGURA 2}

\section{HISTOGRAMA DE LOS EFECTOS FIJOS DE LOS DOCENTES}

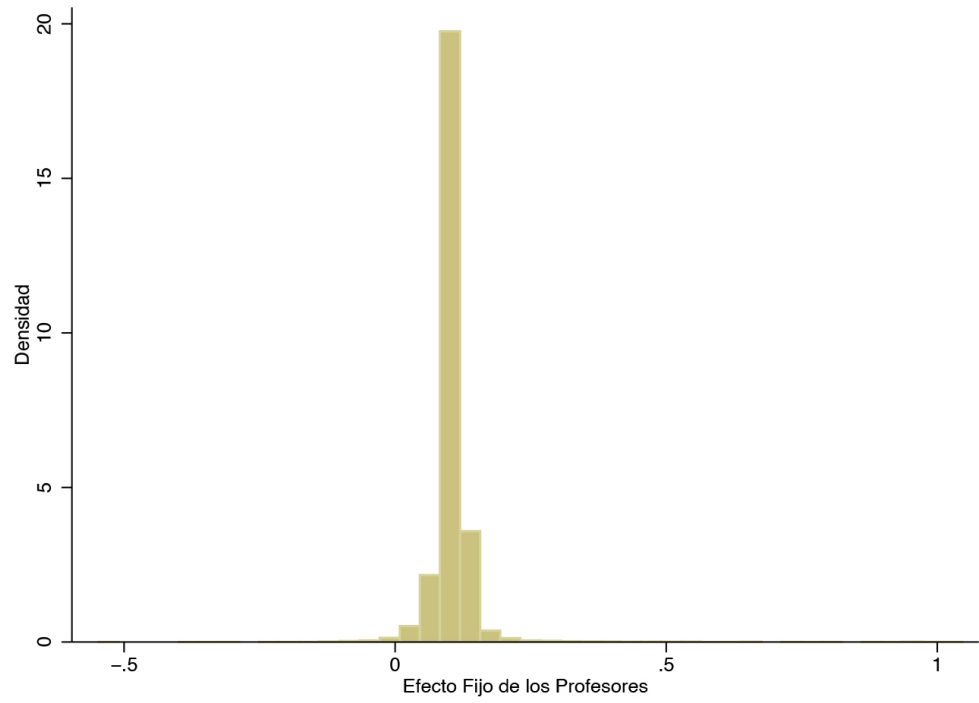

(a) Toda la muestra

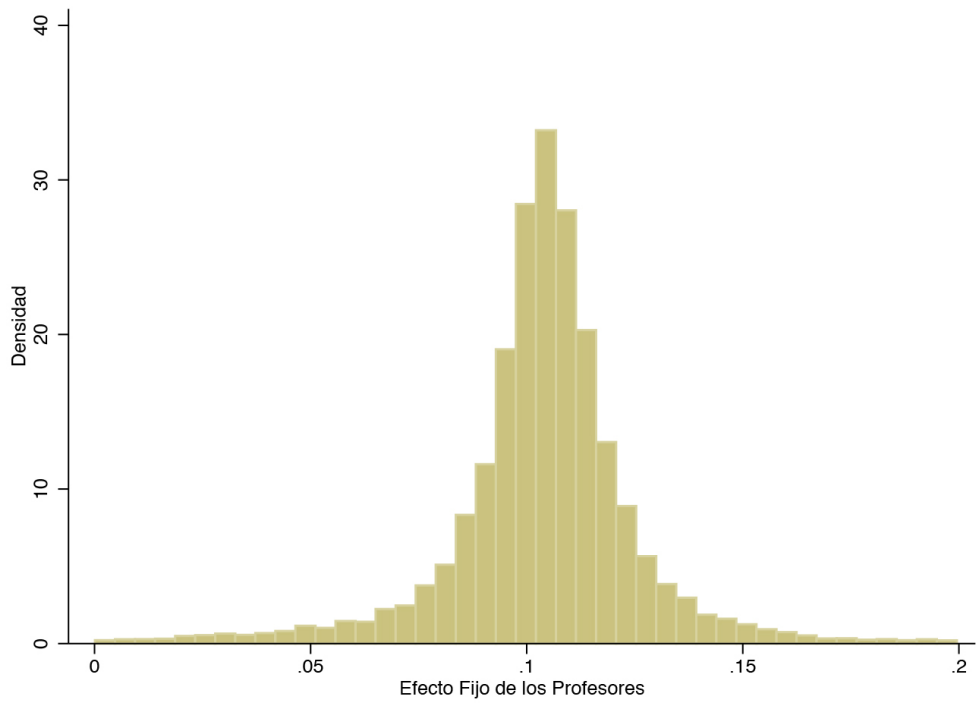

(b) Intervalo $[0,0,2]$

Fuente: Elaboración propia en base a datos de Centro de Estudios, Ministerio de Educación. Chile. 


\section{d) Deserción escolar}

En cuanto a la deserción escolar, Chile es el país que presenta el menor nivel de deserción escolar entre jóvenes de 20 a 24 según un informe elaborado por CEPAL (Trucco y Ullmann, 2015) a nivel latinoamericano tal como se muestra en la Figura 3a. No obstante, si comparamos a Chile con los países más desarrollados el resultado es diferente. Según el estudio de OECD (2007), el mayor impedimento para aumentar la productividad en la economía chilena es el bajo nivel de capital humano en fuerza laboral, el cual está por debajo del promedio de los países que conforman la OECD. Según Lyche (2010), y tal como se muestra en la Figura 3b, la deserción escolar de Chile supera en un 21.6\% a la media del OCDE para jóvenes entre 25 y 34 años.

Según las Encuesta de Caracterización Socioeconómica Nacional (CASEN) del año 2003, la principal razón de la deserción escolar es la paternidad para los jóvenes entre 14 y 17 años. Ellos también mencionan dificultades económicas y de rendimiento académico, en algunos casos tienen empleo y/o falta de interés en asistir a un establecimiento educativo. Para jóvenes entre 18 y 24 años, el trabajo es el principal motivo para desistir del sistema educativo.

En Chile, todas las estadísticas oficiales mencionan la deserción escolar como un porcentaje anual, pero este método no refleja la realidad ya que no podemos comparar alguien que abandona el sistema educativo en los primeros años con un estudiante que lo hace en los últimos años. Es por esto que es mejor observar el fenómeno de la deserción escolar por cohorte, es decir, el porcentaje de alumnos que no logró finalizar la enseñanza secundaria del total de alumnos que entraron al sistema educativo en un año particular.

A continuación, usando el promedio de las cohortes 2002 y $2003^{8}$, mostramos en la Figura 4 que el mayor porcentaje de deserción escolar en Chile se encuentra al finalizar la educación primaria y comenzar la secundaria, produciéndose allí un quiebre importante, ya que en los primeros siete años casi no hay abandono escolar, siendo éste inferior al 6\% acumulado, y hasta el quinto año siendo menor al $3 \%$ acumulado. En cambio, al alcanzar el octavo año de la educación primaria la deserción escolar ya alcanza casi el $8 \%$ acumulado, y luego en el primer año de secundaria llega al $15 \%$ acumulado. Esto nos muestra que no es comparable la deserción escolar en la primaria con la secundaria, por ende no es correcto hablar de "abandono anual", ya que los porcentajes son muy distintos en cada nivel educativo.

8 Las únicas cohortes que observamos en toda su vida escolar. 


\section{FIGURA 3}

\section{COMPARACION DE DESERCION ESCOLAR EN AMERICA LATINA Y PAISES DE LA OECD}

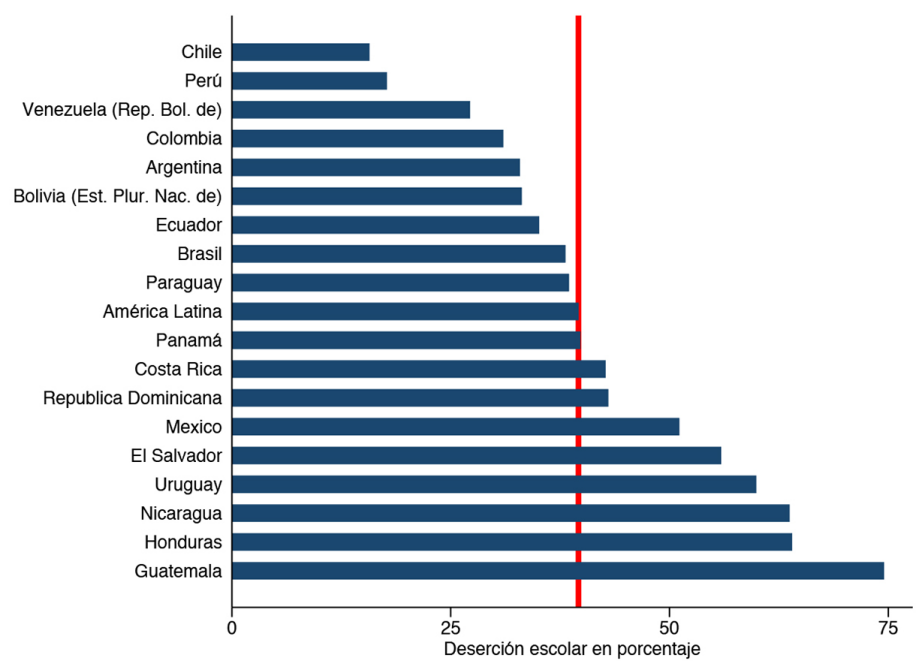

(a) Porcentaje de jóvenes entre 20 y 24 años que no finalizaron la educación secundaria, alrededor del año 2013 para países de América Latina

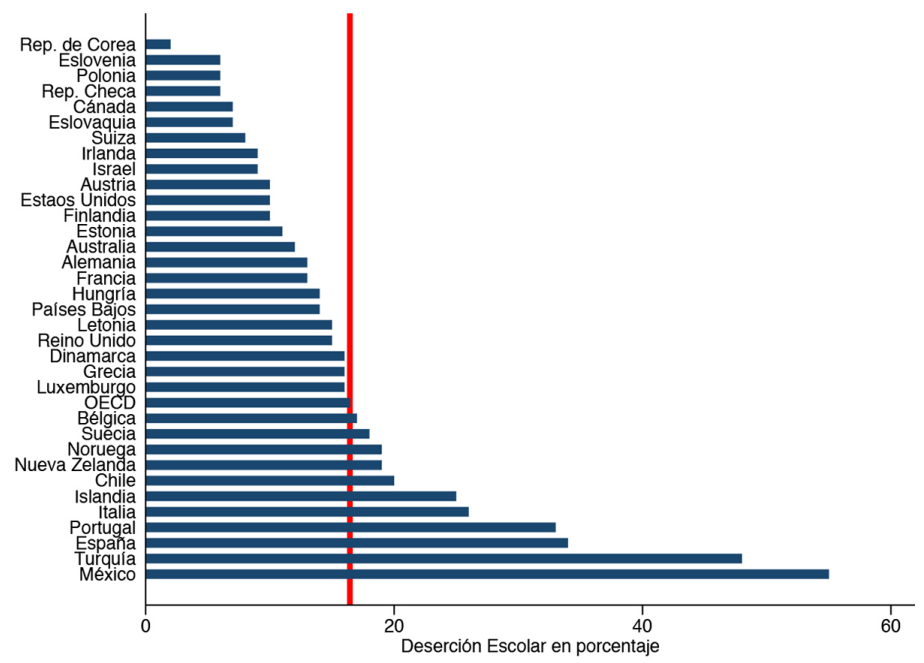

(b) Porcentaje de jóvenes entre 25 y 30 años que no finalizarón la educación secundaria, alrededor del año 2015 para países de la OECD

Fuente: Elaboración propia en base a datos de CEPAL y OECD. 


\section{FIGURA 4}

\section{DESERCION ESCOLAR ACUMULADA DESDE $1^{\circ}$ BASICO}

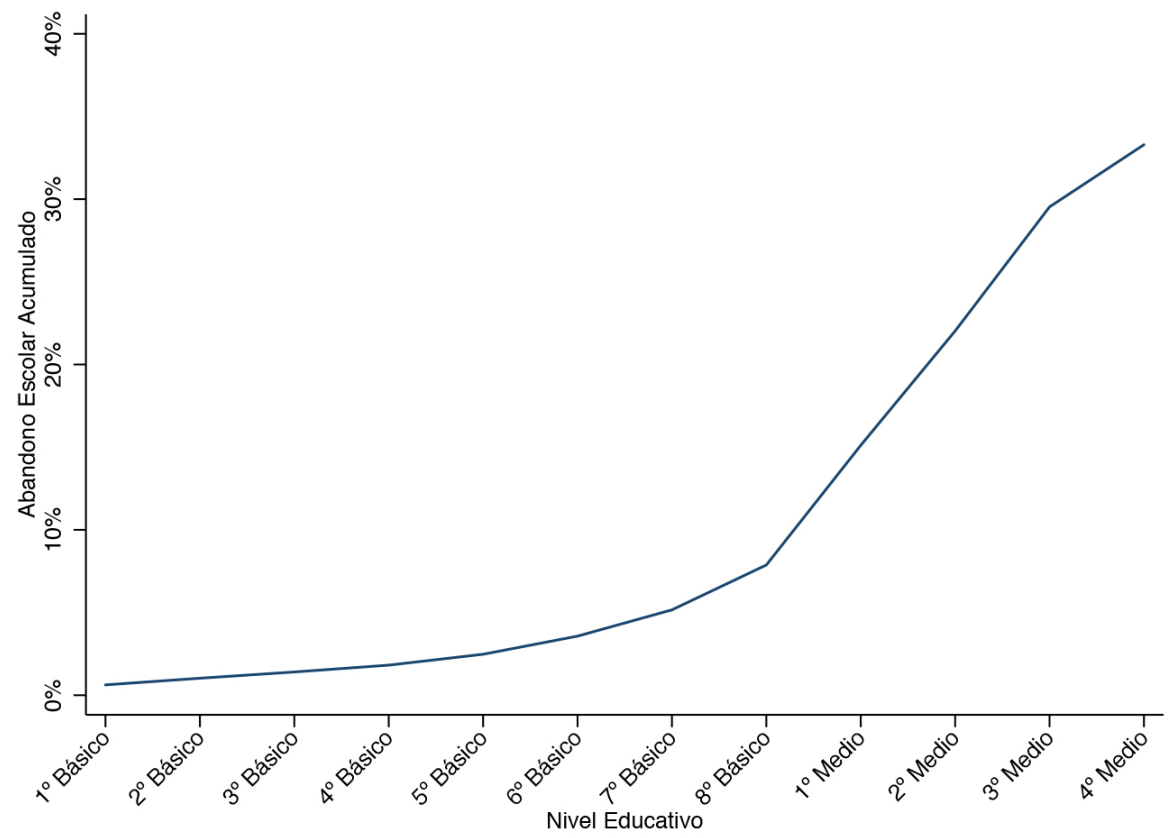

Fuente: Elaboración propia en baso a datos de Centro de Estudios, Ministerio de educación. Chile.

Además, en la Figura 4 se observa que en promedio, de la totalidad de alumnos que ingresaron en los años 2002 y 2003, más del 30\% no concluyó la educación secundaria.

De ahora en adelante, centrándonos únicamente en la educación secundaria, y definiendo como cohorte los jóvenes que ingresaron a la educación secundaria en un año en particular, mostramos en la Tabla 4 la deserción escolar en los alumnos de educación superior para los cohortes desde el año 2002 hasta el 2012, y por cada grado académico, donde se observa claramente que en los tres primeros años es donde se concentra casi la totalidad de la deserción escolar en Chile. Tal como muestra en la Tabla 4, solamente en la educación secundaria, la deserción escolar alcanza en promedio un $25 \%$, pero ya sabemos que si consideramos además los ocho años de escuela primaria, supera el $30 \%$. 


\section{TABLA 4}

\section{DESERCION ESCOLAR EN ALUMNOS DE EDUCACION SECUNDARIA POR COHORTE Y NIVEL EDUCATIVO}

\begin{tabular}{|c|c|c|c|c|c|}
\hline Cohorte & $\begin{array}{c}\text { Deserción } \\
(1)\end{array}$ & $\begin{array}{c}1^{\circ} \text { Medio } \\
(2)\end{array}$ & $\begin{array}{c}2^{\circ} \text { Medio } \\
(3)\end{array}$ & $\begin{array}{c}3^{\circ} \text { Medio } \\
(4)\end{array}$ & $\begin{array}{c}4^{\circ} \text { Medio } \\
(5)\end{array}$ \\
\hline 2002 & $28,90 \%$ & $8,54 \%$ & $9,41 \%$ & $9,26 \%$ & $3,70 \%$ \\
2003 & $25,03 \%$ & $6,61 \%$ & $7,58 \%$ & $8,28 \%$ & $3,58 \%$ \\
2004 & $25,73 \%$ & $6,70 \%$ & $7,90 \%$ & $8,83 \%$ & $3,14 \%$ \\
2005 & $25,64 \%$ & $7,02 \%$ & $8,23 \%$ & $8,13 \%$ & $2,97 \%$ \\
2006 & $25,54 \%$ & $7,47 \%$ & $8,07 \%$ & $7,70 \%$ & $2,94 \%$ \\
2007 & $24,74 \%$ & $7,24 \%$ & $7,28 \%$ & $7,20 \%$ & $3,68 \%$ \\
2008 & $25,20 \%$ & $6,91 \%$ & $6,76 \%$ & $8,11 \%$ & $4,20 \%$ \\
2009 & $25,44 \%$ & $6,79 \%$ & $7,20 \%$ & $8,57 \%$ & $3,47 \%$ \\
2010 & $25,02 \%$ & $7,24 \%$ & $7,48 \%$ & $7,67 \%$ & $3,19 \%$ \\
2011 & $24,90 \%$ & $7,79 \%$ & $7,15 \%$ & $7,30 \%$ & $3,12 \%$ \\
2012 & $23,89 \%$ & $7,08 \%$ & $6,57 \%$ & $8,03 \%$ & $2,94 \%$ \\
\hline
\end{tabular}

Fuente: Elaboración propia en base a datos de Centro de Estudios, Ministerio de Educación. Chile.

A continuación, en la Tabla 5 se presentan estadísticas descriptivas para la deserción escolar por grado y por distintas categorías para las cohortes entre el año 2004 y el año 20119: (i) dependencia administrativa, (ii) sexo del alumno y (iii) ubicación geográfica del establecimiento educativo.

Es fácil notar que la deserción escolar se concentra principalmente en establecimientos municipales (de bajo o nulo costo económico, y de alumnos de clase media baja), rurales y en alumnos hombres en todos los niveles de la educación secundaria.

\section{TABLA 5}

ESTADISTICAS DESCRIPTIVAS DE LA DESERCION ESCOLAR POR NIVEL EDUCATIVO Y CATEGORIA

\begin{tabular}{|l|c|c|c|c|}
\hline Categoría & $\begin{array}{c}1^{\circ} \text { Medio } \\
(1)\end{array}$ & $\begin{array}{c}2^{\circ} \text { Medio } \\
(2)\end{array}$ & $\begin{array}{c}3^{\circ} \text { Medio } \\
(3)\end{array}$ & $\begin{array}{c}4^{\circ} \text { Medio } \\
(4)\end{array}$ \\
\hline $\begin{array}{l}\text { Dependencia administrativa } \\
\text { Municipal }\end{array}$ & $10,27 \%$ & $10,35 \%$ & $10,57 \%$ & $4,65 \%$ \\
Part. Subvencionado & $5,28 \%$ & $6,09 \%$ & $6,84 \%$ & $2,76 \%$ \\
Part. no Subvencionado & $1,25 \%$ & $2,04 \%$ & $2,73 \%$ & $0,86 \%$ \\
Género Alumno & & & & \\
Hombre & $8,39 \%$ & $8,59 \%$ & $9,15 \%$ & $3,68 \%$ \\
Mujer & $5,87 \%$ & $6,45 \%$ & $6,77 \%$ & $3,01 \%$ \\
Ubicación Geográfica & & & & \\
Urbano & $7,03 \%$ & $7,45 \%$ & $7,90 \%$ & $3,31 \%$ \\
Rural & $9,70 \%$ & $9,18 \%$ & $9,33 \%$ & $4,05 \%$ \\
\hline
\end{tabular}

Fuente: Elaboración propia en base a datos de Centro de Estudios, Ministerio de Educación. Chile.

$9 \quad$ Esto debido a que observamos a los alumnos en toda su educación secundaria 


\section{RESULTADOS}

De acuerdo a la estrategia empírica utilizada, con su respectiva especificación econométrica y las variables de interés y de control mencionadas, se obtienen resultados tanto para la probabilidad de deserción escolar como para la movilidad de los profesores. Entre la Tabla 6 y la Tabla 10 se muestran los principales resultados obtenidos en esta investigación.

Primero, al analizar el impacto de los distintos controles en la probabilidad de deserción del alumno se observan impactos positivos y significativos en todos los modelos, en la medida que el alumno es hombre, haya reprobado algún año en la enseñanza básica o haya reprobado anteriormente el nivel de $1^{\circ}$ medio. También se observa una relación negativa para estudiantes de establecimientos particulares subvencionados y no subvencionados, es decir, tienen mayor probabilidad de desertar los alumnos de establecimientos municipales, que, por lo general, son los de menor nivel socioeconómico.

Se estiman tres modelos en la Tabla 6 donde en cada uno se agrega uno de los efectos fijos utilizados. Los resultados muestran que en todos los modelos la mayoría de los signos de los coeficientes y su nivel de significancia permanecen constantes. En particular, los controles por (i) sexo del alumno, (ii) si repitió en la educación primaria, (iii) dependencia administrativa del establecimiento educacional al que asistió el alumno en $1^{\circ}$ medio, y (iv) si ha rendido $1^{\circ}$ medio más de una ocasión ${ }^{10}$ presentan estimadores significativos y con el mismo signo en los tres modelos presentados. Estos resultados son interesantes pues muestran la importancia de la situación socioeconómica de los alumnos a la hora de tomar la decisión de desertar o no del sistema educativo. Además los resultados son consistentes con las estadísticas descriptivas presentadas en la Tabla 5 y los resultados previos de la literatura mencionada; los establecimientos particulares disminuyen el nivel de deserción escolar de sus alumnos, el cual es un fuerte componente socioeconómico.

Se observa que la probabilidad de deserción aumenta de $6.57 \%$ (promedio de los datos ${ }^{11}$ ) a un $6.59 \%$ si el alumno es hombre y a un $12.71 \%$ si repitió algún año escolar en la educación primaria. Además, la probabilidad de deserción escolar aumenta a un $16.39 \%$ si cursa $1^{\circ}$ medio por segunda vez y $32.93 \%$ si cursa $1^{\circ}$ medio por tercera vez ${ }^{12}$.

10 En los datos originales, se encontró que el máximo de veces que un alumno cursó $1^{\circ}$ medio fue seis veces. En la regresión original se han agregado variables binarias para cada caso, pero se han excluido en la presentación de resultados porque no es relevante, ya que para todos los casos se encontró un coeficiente positivo y significativo.

11 La diferencia con la Tabla 3 se debe a que en la regresión se ha usado un panel de datos con base a alumnos por profesor por año.

12 Todos los cálculos fueron realizados con el Modelo 3 de la Tabla 5. 
A continuación, luego de capturar el efecto fijo de los docentes en esta primera estimación, se han estimado cuatro modelos para saber si hay relación entre esta estimación de valor agregado y ciertas características de movilidad de los docentes; donde trabajan, y si tienden a salir o entrar en muchos establecimientos educativos.

Primero, se ha realizado un ranking de los profesores según la magnitud de su efecto fijo, o valor agregado, capturado anteriormente para luego separarlos en cuartiles: El primer cuartil es el de los mejores profesores, o de mayor valor agregado, ya que tienen una menor participación en la deserción escolar de sus alumnos, ellos están dentro del 25\% mejor calificado. Mientras que el cuarto cuartil, es el de los profesores peor calificados o de menor valor agregado, ya que tienen una alta participación en la deserción escolar de sus alumnos, ellos están dentro del $25 \%$ peor calificado.

\section{TABLA 6}

ESTIMACION DE PROBABILIDAD DE DESERCION ESCOLAR

\begin{tabular}{|c|c|c|c|}
\hline Variables & $\begin{array}{c}\text { (1) } \\
\text { Modelo } 1\end{array}$ & $\begin{array}{c}\text { (2) } \\
\text { Modelo } 2\end{array}$ & $\begin{array}{c}\text { (3) } \\
\text { Modelo } 3\end{array}$ \\
\hline Hombre & $\begin{array}{c}0,0087 * * * \\
(0,0002)\end{array}$ & $\begin{array}{c}0,0080 * * * \\
(0,0002)\end{array}$ & $\begin{array}{c}0,0002 * * * \\
(0,0001)\end{array}$ \\
\hline Repitió en educación primaria & $\begin{array}{c}0,1568 * * * \\
(0,0004)\end{array}$ & $\begin{array}{c}0,1476^{* * * *} \\
(0,0004)\end{array}$ & $\begin{array}{c}0,0614 * * * \\
(0,0001)\end{array}$ \\
\hline Se cambió de establecimiento en $8^{\circ}$ básico & $\begin{array}{c}0,0176^{* * * *} \\
(0,0002)\end{array}$ & $\begin{array}{c}0,0158 * * * * \\
(0,0002)\end{array}$ & $\begin{array}{c}-0,1134 * * * \\
(0,0001)\end{array}$ \\
\hline Establecimiento rural en $8^{\circ}$ básico & $\begin{array}{c}0,0085^{\text {**** }} \text { * } \\
(0,0005)\end{array}$ & $\begin{array}{c}0,0066^{* * * *} \\
(0,0010)\end{array}$ & $\begin{array}{c}-0,0027 * * * \\
(0,0002)\end{array}$ \\
\hline Segunda vez cursando $1^{\circ}$ medio & $\begin{array}{c}0,1855^{* * * *} * \\
(0,0005)\end{array}$ & $\begin{array}{c}0,1729 * * * \\
(0,0005)\end{array}$ & $\begin{array}{c}0,0982 * * * * \\
(0,0001)\end{array}$ \\
\hline Tercera vez cursando $1^{\circ}$ medio & $\begin{array}{c}0,4277 * * * \\
(0,0016)\end{array}$ & $\begin{array}{c}0,4043 * * * \\
(0,0016)\end{array}$ & $\begin{array}{c}0,2636 * * * \\
(0,0004)\end{array}$ \\
\hline Constante & $\begin{array}{c}0,0280^{* * * *} \\
(0,0003)\end{array}$ & $\begin{array}{c}0,0338 * * * \\
(0,0005)\end{array}$ & $\begin{array}{c}0,0000 \\
(0,0003)\end{array}$ \\
\hline Promedio de deserción escolar & 0,0657 & 0,0657 & 0,0657 \\
\hline Observaciones & 6.307 .167 & 6.307 .167 & 5.901 .679 \\
\hline $\mathrm{R}$-squared & 0,1644 & 0,1816 & 0,7065 \\
\hline EF por años & SI & SI & SI \\
\hline EF por Ranking y Establecimiento $8^{\circ}$ Básico & $\mathrm{NO}$ & NO & SI \\
\hline EF por profesor & NO & SI & SI \\
\hline
\end{tabular}

Notas: Errores estándar robustos a heterosedasticidad en paréntesis. *** $\mathrm{p}<0,01, * * \mathrm{p}<0,05, * \mathrm{p}<0,1$. 
En el primer modelo, estimado en la Tabla 7, se observa la estimación de probabilidad de que un profesor realice clases en cada tipo de establecimiento. Los resultados muestran que los profesores de menor valor agregado (cuarto cuartil) tienen mayores probabilidades de trabajar en establecimientos municipales en comparación de los profesores de mayor valor agregado (primer cuartil y categoría omitida para evitar colinealidad perfecta). Por otro lado, los profesores de los cuartiles intermedios, tienen menos probabilidades de trabajar en establecimientos municipales, pero más probabilidades de trabajar en establecimientos particulares. Finalmente, los profesores de menor valor agregado menores probabilidades de trabajar en establecimientos particulares subvencionados y no subvencionados que los profesores de mayor valor agregado.

\section{TABLA 7}

ESTIMACION DE PROBABILIDAD DE REALIZAR CLASES EN CADA TIPO DE ESTABLECIMIENTO EDUCATIVO

\begin{tabular}{|c|c|c|c|}
\hline Variables & $\begin{array}{c}(1) \\
\text { Municipal }\end{array}$ & $\begin{array}{c}(2) \\
\text { Particular } \\
\text { Subvencionado }\end{array}$ & $\begin{array}{c}\text { (3) } \\
\text { Particular no } \\
\text { Subvencionado }\end{array}$ \\
\hline Segundo Cuartil & $\begin{array}{c}-0,0942 * * * \\
(0,0034)\end{array}$ & $\begin{array}{c}0,0106 * * * \\
(0,0035)\end{array}$ & $\begin{array}{c}0,0686 * * * \\
(0,0025)\end{array}$ \\
\hline Tercer Cuartil & $\begin{array}{c}-0,0779 * * * \\
(0,0034)\end{array}$ & $\begin{array}{c}0,0106 * * * \\
(0,0035)\end{array}$ & $\begin{array}{c}0,0549 * * * \\
(0,0025)\end{array}$ \\
\hline Cuarto Cuartil & $\begin{array}{c}0,0282 * * * \\
(0,0036)\end{array}$ & $\begin{array}{c}-0,0290 * * * \\
(0,0037)\end{array}$ & $\begin{array}{c}-0,0077 * * * \\
(0,0023)\end{array}$ \\
\hline Estudios Presenciales & $\begin{array}{c}-0,1336 * * * \\
(0,0129)\end{array}$ & $\begin{array}{c}0,1271 * * * \\
(0,0133)\end{array}$ & $\begin{array}{l}-0,0027 \\
(0,0076)\end{array}$ \\
\hline Estudios Universitarios & $\begin{array}{c}0,0771 * * * \\
(0,0104)\end{array}$ & $\begin{array}{c}-0,0766 * * * \\
(0,0106)\end{array}$ & $\begin{array}{c}0,0236 * * * \\
(0,0065)\end{array}$ \\
\hline Experiencia & $\begin{array}{c}0,0065 * * * \\
(0,0005)\end{array}$ & $\begin{array}{c}-0,0115 * * * \\
(0,0005)\end{array}$ & $\begin{array}{c}0,0106 * * * \\
(0,0004)\end{array}$ \\
\hline Experiencia $^{2}$ & $\begin{array}{l}-0,0000 \\
(0,0000)\end{array}$ & $\begin{array}{c}0,0001 * * * \\
(0,0000)\end{array}$ & $\begin{array}{c}-0,0002 * * * \\
(0,0000)\end{array}$ \\
\hline Edad & $\begin{array}{c}0,0048 * * * \\
(0,0003)\end{array}$ & $\begin{array}{c}-0,0022 * * * \\
(0,0003)\end{array}$ & $\begin{array}{c}-0,0033 * * * \\
(0,0002)\end{array}$ \\
\hline Profesor Hombre & $\begin{array}{c}0,0197 * * * \\
(0,0025)\end{array}$ & $\begin{array}{c}0,0266 * * * \\
(0,0026)\end{array}$ & $\begin{array}{c}0,0213 * * * \\
(0,0019)\end{array}$ \\
\hline Constante & $\begin{array}{c}0,2237 * * * \\
(0,0144)\end{array}$ & $\begin{array}{c}0,6827 * * * \\
(0,0150)\end{array}$ & $\begin{array}{c}0,1409 * * * \\
(0,0088)\end{array}$ \\
\hline Promedio de los datos & 0,3712 & 0,5264 & 0,1465 \\
\hline Observaciones & 156.848 & 156.848 & 156.848 \\
\hline R-squared & 0,0750 & 0,0584 & 0,0191 \\
\hline Efectos fijos por año & SI & SI & SI \\
\hline
\end{tabular}

Notas: Errores estándar robustos a heterosedasticidad en paréntesis. *** $\mathrm{p}<0,01, * * \mathrm{p}<0,05, * \mathrm{p}<0,1$. 


\section{TABLA 8}

\section{ESTIMACION DE PROBABILIDAD DE REALIZAR CLASES \\ EN CADA TIPO DE ESTABLECIMIENTO EDUCATIVO, DADO QUE SOLO HACE CLASES EN ESE TIPO DE ESTABLECIMIENTO}

\begin{tabular}{|c|c|c|c|}
\hline Variables & $\begin{array}{c}\text { (1) } \\
\text { Municipal }\end{array}$ & $\begin{array}{c}(2) \\
\text { Particular } \\
\text { Subvencionado }\end{array}$ & $\begin{array}{c}\text { (3) } \\
\text { Particular no } \\
\text { Subvencionado }\end{array}$ \\
\hline Segundo Cuartil & $\begin{array}{c}-0,0860 * * * \\
(0,0033)\end{array}$ & $\begin{array}{c}0,0159^{* * * *} \\
(0,0035)\end{array}$ & $\begin{array}{c}0,0703 * * * \\
(0,0024)\end{array}$ \\
\hline Tercer Cuartil & $\begin{array}{c}-0,0737 \text { *** } \\
(0,0033)\end{array}$ & $\begin{array}{c}0,0148 \text { *** } \\
(0,0035)\end{array}$ & $\begin{array}{c}0,0534 * * * \\
(0,0023)\end{array}$ \\
\hline Cuarto Cuartil & $\begin{array}{c}0,0244 * * * \\
(0,0035)\end{array}$ & $\begin{array}{c}-0,0311 * * * \\
(0,0036)\end{array}$ & $\begin{array}{c}-0,0044 * * \\
(0,0022)\end{array}$ \\
\hline Estudios Presenciales & $\begin{array}{c}-0,1139 \text { *** } \\
(0,0125)\end{array}$ & $\begin{array}{c}0,1419 \text { *** } \\
(0,0131)\end{array}$ & $\begin{array}{c}0,0414 * * * \\
(0,0068)\end{array}$ \\
\hline Estudios Universitarios & $\begin{array}{c}0,0600 * * * \\
(0,0099)\end{array}$ & $\begin{array}{c}-0,0935^{* * * *} \\
(0,0106)\end{array}$ & $\begin{array}{c}0,0267 * * * * \\
(0,0060)\end{array}$ \\
\hline Experiencia & $\begin{array}{c}0,0029 * * * \\
(0,0004)\end{array}$ & $\begin{array}{c}-0,0147 * * * \\
(0,0005)\end{array}$ & \\
\hline Experiencia $^{2}$ & $\begin{array}{c}0,0000^{* * * *} \\
(0,0000)\end{array}$ & $\begin{array}{c}0,0001 * * * * \\
(0,0000)\end{array}$ & $\begin{array}{c}0,0000^{* * * *} \\
(0,0000)\end{array}$ \\
\hline Edad & $\begin{array}{c}0,0048 * * * \\
(0,0002)\end{array}$ & $\begin{array}{c}-0,0024 * * * \\
(0,0003)\end{array}$ & $\begin{array}{c}-0,0007 * * * \\
(0,0001)\end{array}$ \\
\hline Profesor Hombre & $\begin{array}{c}-0,0341 * * * \\
(0,0024)\end{array}$ & $\begin{array}{c}-0,0300 * * * \\
(0,0025)\end{array}$ & $\begin{array}{c}0,0000 \\
(0,0018)\end{array}$ \\
\hline Constante & $\begin{array}{c}0,1665^{* * * *} \\
(0,0139)\end{array}$ & $\begin{array}{c}0,6267 * * * * \\
(0,0150)\end{array}$ & $\begin{array}{c}0,0529^{* * * *} \\
(0,0080)\end{array}$ \\
\hline Promedio de los datos & 0,3079 & 0,4593 & 0,1282 \\
\hline Observaciones & 156.848 & 156.848 & 156.848 \\
\hline $\mathrm{R}$-squared & 0,0536 & 0,0827 & 0,0115 \\
\hline Efectos fijos por año & SI & SI & SI \\
\hline
\end{tabular}

Notas: Errores estándar robustos a heterosedasticidad en paréntesis. *** $\mathrm{p}<0,01, * * \mathrm{p}<0,05, * \mathrm{p}<0,1$.

A continuación, en la Tabla 8 se muestran los resultados del segundo modelo de esta sección, donde la variable dependiente es la probabilidad de hacer clases en cada tipo de establecimiento educativo, pero únicamente en ese tipo de establecimiento. Mostrando resultados consistentes y similares con los estimados en la Tabla 7. Los profesores de menor valor agregado tienen menos probabilidades de hacer clases en un establecimiento particular subvencionado y particular no subvencionado, pero tienen más probabilidades de estar en establecimientos municipales. 
Por otro lado, en el tercer modelo de esta sección estimado en la Tabla 9, se estima la probabilidad de que un profesor siga haciendo clases en un tipo de establecimiento, dado que ya hacia clases en ese mismo tipo el año anterior, donde se observa que los profesores de menor valor agregado tienen más probabilidades de permanecer en un establecimiento municipal, pero menores posibilidades de permanecer en un establecimiento particular.

\section{TABLA 9}

\section{PROBABILIDAD DE REALIZAR CLASES EN CADA TIPO DE ESTABLECIMIENTO EDUCATIVO, DADO QUE HACIA CLASES EN EL MISMO TIPO DE ESTABLECIMIENTO EL AÑO ANTERIOR}

\begin{tabular}{|c|c|c|c|}
\hline Variables & $\begin{array}{c}\text { (1) } \\
\text { Municipal }\end{array}$ & $\begin{array}{c}(2) \\
\text { Particular } \\
\text { Subvencionado }\end{array}$ & $\begin{array}{c}\text { (3) } \\
\text { Particular no } \\
\text { Subvencionado }\end{array}$ \\
\hline Segundo Cuartil & $\begin{array}{c}-0,0691 * * * \\
(0,0031)\end{array}$ & $\begin{array}{c}0,0286 * * * \\
(0,0035)\end{array}$ & $\begin{array}{c}0,0566 * * * \\
(0,0022)\end{array}$ \\
\hline Tercer Cuartil & $\begin{array}{c}-0,0559 * * * \\
(0,0031)\end{array}$ & $\begin{array}{c}0,0274 * * * \\
(0,0035)\end{array}$ & $\begin{array}{c}0,0470 * * * \\
(0,0022)\end{array}$ \\
\hline Cuarto Cuartil & $\begin{array}{c}0,0270 * * * * \\
(0,0033)\end{array}$ & $\begin{array}{c}-0,0142 * * * \\
(0,0036)\end{array}$ & $\begin{array}{c}-0,0049 * * \\
(0,0021)\end{array}$ \\
\hline Estudios Presenciales & $\begin{array}{c}-0,1378 * * * \\
(0,0119)\end{array}$ & $\begin{array}{c}0,1215^{* * * *} \\
(0,0132)\end{array}$ & $\begin{array}{c}-0,0143^{* *} \\
(0,0069)\end{array}$ \\
\hline Estudios Universitarios & $\begin{array}{c}0,0831 * * * \\
(0,0096)\end{array}$ & $\begin{array}{c}-0,0685 * * * \\
(0,0104)\end{array}$ & $\begin{array}{c}0,0148 * * \\
(0,0059)\end{array}$ \\
\hline Experiencia & $\begin{array}{c}0,0110 * * * \\
(0,0004)\end{array}$ & $\begin{array}{c}0,0028 * * * * \\
(0,0005)\end{array}$ & $\begin{array}{c}0,0111 * * * \\
(0,0003)\end{array}$ \\
\hline Experiencia $^{2}$ & $\begin{array}{c}-0,0001 * * * \\
(0,0000)\end{array}$ & $\begin{array}{c}-0,0003 * * * \\
(0,0000)\end{array}$ & $\begin{array}{c}-0,0002 * * * \\
(0,0000)\end{array}$ \\
\hline Edad & $\begin{array}{c}0,0052 * * * \\
(0,0002)\end{array}$ & $\begin{array}{c}0,0004 \\
(0,0003)\end{array}$ & $\begin{array}{c}-0,0022 * * * \\
(0,0002)\end{array}$ \\
\hline Profesor Hombre & $\begin{array}{c}0,0121^{* * *} * \\
(0,0023)\end{array}$ & $\begin{array}{c}0,0190 * * * \\
(0,0025)\end{array}$ & $\begin{array}{c}0,0176 * * * \\
(0,0017)\end{array}$ \\
\hline Constante & $\begin{array}{c}-0,2795 * * * \\
(0,0120)\end{array}$ & $\begin{array}{c}-0,0513 * * * \\
(0,0142)\end{array}$ & $\begin{array}{c}-0,0536 * * * \\
(0,0069)\end{array}$ \\
\hline Promedio de los datos & 0,2916 & 0,4027 & 0,1115 \\
\hline Observaciones & 156.848 & 156.848 & 156.848 \\
\hline R-squared & 0,1253 & 0,0662 & 0,0310 \\
\hline Efectos fijos por año & SI & SI & SI \\
\hline
\end{tabular}

Notas: Errores estándar robustos a heterosedasticidad en paréntesis. ${ }^{* * *} \mathrm{p}<0,01, * * \mathrm{p}<0,05,{ }^{*} \mathrm{p}<0,1$. 
Finalmente, en el último modelo de esta sección se estima la probabilidad de que un profesor abandone un establecimiento educativo de cada tipo, donde los resultados se muestran en la Tabla 10. Se observa que mientras peor sea el valor agregado de los profesores, mayores probabilidades tiene de abandonar el establecimiento de tipo particular no subvencionado. Por otro lado los profesores de menor valor agregado, tienen menos posibilidades de abandonar establecimientos municipales y particulares subvencionados.

\section{TABLA 10}

ESTIMACION DE PROBABILIDAD DE ABANDONAR CADA TIPO DE ESTABLECIMIENTO EDUCATIVO

\begin{tabular}{|c|c|c|c|}
\hline Variables & $\begin{array}{c}\text { (1) } \\
\text { Municipal }\end{array}$ & $\begin{array}{c}\text { (2) } \\
\text { Particular } \\
\text { Subvencionado }\end{array}$ & $\begin{array}{c}\text { (3) } \\
\text { Particular no } \\
\text { Subvencionado }\end{array}$ \\
\hline Segundo Cuartil & $\begin{array}{c}-0,0022^{* * *} \\
(0,0009)\end{array}$ & $\begin{array}{l}-0,0006 \\
(0,0011)\end{array}$ & $\begin{array}{c}0,0018^{* * * *} \\
(0,0007)\end{array}$ \\
\hline Tercer Cuartil & $\begin{array}{l}-0,0013 \\
(0,0009)\end{array}$ & $\begin{array}{l}-0,0012 \\
(0,0011)\end{array}$ & $\begin{array}{c}0,0023 * * * \\
(0,0007)\end{array}$ \\
\hline Cuarto Cuartil & $\begin{array}{l}-0,0001 \\
(0,0010)\end{array}$ & $\begin{array}{l}-0,0012 \\
(0,0012)\end{array}$ & $\begin{array}{c}0,0001 \\
(0,0007)\end{array}$ \\
\hline Estudios Presenciales & $\begin{array}{c}0,0094 * * * \\
(0,0034)\end{array}$ & $\begin{array}{c}0,0145 * * * \\
(0,0033)\end{array}$ & $\begin{array}{c}0,0040 * * \\
(0,0018)\end{array}$ \\
\hline Estudios Universitarios & $\begin{array}{l}-0,0019 \\
(0,0026)\end{array}$ & $\begin{array}{c}0,0108 * * * \\
(0,0026)\end{array}$ & $\begin{array}{l}0,0027 * \\
(0,0015)\end{array}$ \\
\hline Experiencia & $\begin{array}{c}-0,0018 * * * \\
(0,0001)\end{array}$ & $\begin{array}{c}-0,0009 * * * \\
(0,0002)\end{array}$ & $\begin{array}{c}-0,0002^{* *} \\
(0,0001)\end{array}$ \\
\hline Experiencia $^{2}$ & $\begin{array}{c}0,0000 * * * \\
(0,0000)\end{array}$ & $\begin{array}{c}0,0000 * * * \\
(0,0000)\end{array}$ & $\begin{array}{c}0,0000 \\
(0,0000)\end{array}$ \\
\hline Edad & $\begin{array}{l}-0,0000 \\
(0,0001)\end{array}$ & $\begin{array}{l}-0,0001 \\
(0,0001)\end{array}$ & $\begin{array}{l}-0,0001 \\
(0,0001)\end{array}$ \\
\hline Profesor Hombre & $\begin{array}{c}0,0061^{* * *} * \\
(0,0007)\end{array}$ & $\begin{array}{c}0,0073 * * * \\
(0,0008)\end{array}$ & $\begin{array}{c}0,0024 * * * \\
(0,0005)\end{array}$ \\
\hline Constante & $\begin{array}{c}0,0291 * * * \\
(0,0045)\end{array}$ & $\begin{array}{c}0,0197 * * * \\
(0,0043)\end{array}$ & $\begin{array}{c}0,0119 * * * \\
(0,0026)\end{array}$ \\
\hline Promedio de los datos & 0,0162 & 0,0253 & 0,0091 \\
\hline Observaciones & 156,848 & 156,848 & 156,848 \\
\hline $\mathrm{R}$-squared & 0,0064 & 0,0056 & 0,0022 \\
\hline Efectos fijos por año & SI & SI & SI \\
\hline
\end{tabular}

Notas: Errores estándar robustos a heterosedasticidad en paréntesis. *** $\mathrm{p}<0,01, * * \mathrm{p}<0,05, * \mathrm{p}<0.1$. 


\section{CONCLUSIONES}

Uno de los principales focos de interés en temas de educación es la deserción escolar que afecta a miles de niños y jóvenes en Chile. Si bien existe una vasta literatura que abarca este tema en el país, la mayoría de esta lo hace centrándose únicamente en factores socioeconómicos debido a que sus fuentes de datos son encuestas de hogares. Este trabajo es una primera aproximación para relacionar la deserción escolar con la calidad de sus profesores.

Como forma de análisis, este estudio ha explotado la idea de que el efecto fijo de los profesores, al momento de estimar la probabilidad de deserción escolar de sus alumnos, es una buena aproximación de su "calidad" o de su "valor agregado", y por ende nos puede explicar cómo se mueven los distintos docentes entre los establecimientos educativos.

Gran parte de esta investigación se ha centrado en buscar posibles causantes de la deserción escolar en alumnos de primer año de educación secundaria. En este sentido, se ha encontrado evidencia significativa de que ser hombre, haber reprobado algún año académico en la educación primaria o incluso haber reprobado su primer año de secundaria, aumenta las posibilidades de deserción escolar de los alumnos una vez que se controla por efectos fijos de sus profesores y el establecimiento de donde el alumno finalizó su educación primaria. Además se ha encontrado evidencia significativa, que haber cursado su primer año de educación primaria en un establecimiento particular reduce las probabilidades de deserción.

Posteriormente, usando el efecto fijo capturado como un instrumento para medir la "calidad docente" o el "valor agregado" de los profesores, buscamos los posibles impactos de este índice en características de movilidad de los mismos. Encontramos evidencia de que los profesores con menor índice de calidad, es decir, de menor valor agregado, tienen más posibilidades de trabajar en establecimientos municipales, que son de bajo costo económico, y a la vez tienen menos posibilidades de trabajar en establecimientos particulares, que son altamente costosos en Chile. Además se muestra que los profesores de menor valor agregado tienden a tener mayores posibilidades de abandonar establecimientos particulares, pero tienen mayores posibilidades de quedarse en establecimientos municipales.

Finalmente, y de acuerdo a la estrategia empírica utilizada y los modelos econométricos especificados, este trabajo ha encontrado evidencia que demuestra que los profesores tienen incidencia relevante en la decisión de deserción de sus alumnos, y que esta variable es un buen índice de su calidad como docente, ya que puede explicar en qué tipo de establecimientos educacionales están trabajando, si se quedan o si se cambian de tipo de establecimiento. 


\section{BIBLIOGRAFIA}

BARRO, R. J. (1991). "Economic Growth in a Cross Section of Countries", The Quarterly Journal of Economics, 106 (2), pp. 407-433.

BEYER, H. (1998). “¿Desempleo Juvenil o un Problema de Deserción Escolar?”, Centro de Estudios Públicos, 71, pp. 89-119.

CANALES, A. y MALDONADO, L. (2018). "Teacher quality and student achievement in Chile: Linking teachers' contribution and observable characteristics", International Journal of Educational Development, 60, pp. 33-50.

CAVICCHIOLO, E.; ALIVERNINI, F. y MANGANELLI, S. (2015). "A Mixed Method Study on Teachers' Diaries: Teachers' Narratives and Value-added Patterns", Procedia - Social and Behavioral Sciences, 205, pp. 485-492.

CEPAL. (2002). Panorama social de América Latina: 2001-2002. Comisión Económica para América Latina y el Caribe, Publicación de las Naciones Unidas, Santiago.

CHETTY, R.; FRIEDMAN, J. N. y ROCKOFF, J. E. (2014a). "Measuring the Impacts of Teachers I: Evaluating Bias in Teacher Value-Added Estimates", American Economic Review, 104 (9), pp. 2593-2632.

CHETTY, R.; FRIEDMAN, J. N. y ROCKOFF, J. E. (2014b). "Measuring the Impacts of Teachers II: Teacher Value-Added and Student Outcomes in Adulthood", American Economic Review, 104 (9), pp. 2633-2679.

FILGUEIRA, F.; FUENTES, A., y FILGUEIRA, C. (2001). "Critical Choices at a Critical Age: Youth Emancipation Paths and School Attainment in Latin America", IDB Working Paper, $\mathrm{N}^{\circ} 145$.

HILL, H. C.; KAPITULA, L., y UMLAND, K. (2011). "A Validity Argument Approach to Evaluating Teacher Value-Added Scores", American Educational Research Journal, 48 (3), pp. 794-831.

KOEDEL, C.; MIHALY, K., y ROCKOFF, J. E. (2015). "Value-added modeling: A review", Economics of Education Review, 47, pp. 180-195.

LYCHE, C. (2010). "Taking on the Completion Challenge: A Literature Review on Policies to Prevent Dropout and Early School Leaving”, OECD Education Working Papers, $\mathrm{N}^{\circ} 53$.

MANKIW, N. G.; ROMER, D., \& WEIL, D. N. (1992). "A Contribution to the Empirics of Economic Growth", Quarterly Journal of Economics, 107 (2), pp. 407-437.

MELIS, F.; DIAZ, R. y PALMA, A. (2005). Adolescentes y jóvenes que abandonan sus estudios antes de finalizar la enseñanza media: Principales tendencias, División Social MIDEPLAN, Santiago, Chile.

MONTERO, R. (2007). “Trabajo y deserción escolar: ¿quién protesta por ellos?”, Cuadernos de Economía, 44, pp. 211-231.

OECD. (2007). OECD Economic Survey of Chile. OECD publishing, Paris.

RODRIGUEZ, P.; TRUFFELLO, R.; SUCHAN, K.; VARELA, F.; MATAS, M.; MONDACA, J.; CESPEDES, J.; VALENZUELA, L.; VALENZUELA, J. y ALLENDE GONZALEZ, C. (2016). "Apoyando la formulación de políticas públicas y toma de decisiones en educación utilizando técnicas de análisis de datos masivos: el caso de Chile”, Repositorio Ministerio de Educación de Perú. 18 Diciembre de 2019. <http://repositorio.minedu.gob.pe/handle/123456789/4463>.

SANTOS, H. (2009). "Dinámica de la Deserción Escolar en Chile". Documento de Trabajo Centro de Políticas Comparadas en Educación No 3, Universidad Diego Portales, Santiago. 19 de Diciembre 2019

SAPELLI, C. y TORCHE, A. (2004). "Deserción Escolar y Trabajo Juvenil: ¿Dos Caras de Una Misma Decisión?", Cuadernos de economía, 41 (123), pp. 173-198.

SASS, T. R.; SEMYKINA, A., \& HARRIS, D. N. (2014). "Value-added models and the measurement of teacher productivity", Economics of Education Review, 38, pp. 9-23.

TRUCCO, D. y H. ULLMANN (2016). Juventud: Realidades y Retos para un Desarrollo con Igualdad, Comisión Económica para América Latina y el Caribe, Publicación de las Naciones Unidas, Santiago. 
